\title{
RADIATION CHARGE EXCHANGE AND RADIATION ION-ATOM RECOMBINATION AS A SOURCE OF CONTINUAL
}

\section{E-M RADIATION FROM ASTROPHYSICAL PLASMA}

\author{
A.A. MIHAJLOV \\ Institute of Physics, P.O.Box 57, 11001 Beograd, Yugoslavia \\ M.S. DIMITRIJEVIC \\ Astronomical Observatory, Volgina 7, 11050 Beograd, Yugoslavia \\ and \\ A.M. ERMOLAEV \\ Departement of Physics, University of Durham, Science Laboratories, \\ Durham DH1 3LE, UK
}

We show that for the study of emission from weakly ionized low temperature hydrogen plasmas, the processes $A^{+}+A \rightarrow A^{+}+A+\hbar \omega$ and $A^{+}+A \rightarrow A_{2}^{+}+\hbar \omega$ (where A denotes a neutral atom in ground state and, $A^{+}$and $A_{2}^{+}$atomic and molecular ions) must be treaded as a source of continual electro-magnetic radiation from low temperature plasma. Both reaction channels are treated separately and the corresponding total and separate spectral intensities are determined for hydrogen plasma at $T<10000 \mathrm{~K}$. The obtained results have been also compared with the corresponding spectral intensities for electron-ion bremsstrahlung and electron-ion photorecombination.

Our results (which will be published in Mihajlov et al.,1992) show that in the case of low temperature plasma one must particularly be carefull concerning the continuous EM-radiation spectrum nature. Namely, at typical values of electron and atom component ratio in hydrogen plasma, investigated ion-atom radiation processes might completly determine the character of spontaneous EM-radiation spectrum. We expect similar results in the case of helium plasma. If this fact is not taken into account, serious errors in plasma diagnostic might follow. The important astrophysical cases of interest are hydrogen clouds, circumstellar hydrogen shells and e.g. solar photosphere and chromosphere.

\section{References}

Mihajlov, A.A., Dimitrijevic, M.S., and Ermolaev, A.M. (1992), in press. 